\title{
Strong spin-orbit fields and Dyakonov-Perel spin dephasing in supported metallic films
}

\author{
Nguyen H. Long, ${ }^{*}$ Phivos Mavropoulos, ${ }^{\dagger}$ David S. G. Bauer, Bernd Zimmermann, Yuriy Mokrousov, ${ }^{\ddagger}$ and Stefan Blügel \\ Peter Grünberg Institut and Institute for Advanced Simulation, Forschungszentrum Jülich and JARA, D-52425 Jülich, Germany \\ (Received 6 April 2016; revised manuscript received 12 September 2016; published 22 November 2016)
}

\begin{abstract}
Spin dephasing by the Dyakonov-Perel mechanism in metallic films deposited on insulating substrates is revealed, and quantitatively examined by means of density functional calculations combined with a kinetic equation. The surface-to-substrate asymmetry, probed by the metal wave functions in thin films, is found to produce strong spin-orbit fields and a fast Larmor precession, giving a dominant contribution to spin decay over the Elliott-Yafet spin relaxation up to a thickness of $70 \mathrm{~nm}$. The spin dephasing is oscillatory in time with a rapid (subpicosecond) initial decay. However, parts of the Fermi surface act as spin traps, causing a persistent tail signal lasting 1000 times longer than the initial decay time. It is also found that the decay depends on the direction of the initial spin polarization, resulting in a spin-dephasing anisotropy of $200 \%$ in the examined cases.
\end{abstract}

DOI: 10.1103/PhysRevB.94.180406

In spintronics experiments, spins are often excited in, or transported through, nonmagnetic metallic thin film media [1]. Typical examples are $\mathrm{Cu}, \mathrm{Au}$, or $\mathrm{Pt}$, used in spin-current creation or detection via the spin Hall effect [2-5] or spin Nernst effect [6,7]. Paramount for the spin-transport properties of a medium is the characteristic time $T$ after which the outof-equilibrium spin population that was created in the medium is lost by relaxation or dephasing [8,9]. The microscopic mechanisms leading to spin reduction depend on the material properties, and it is commonly accepted that the Elliott-Yafet (EY) mechanism [10,11] is dominant in metals [12-15], since they show space-inversion symmetry [16]. However, any substrate on which the film is deposited breaks the inversion symmetry; if the film is thin enough (thinner than the electron phase relaxation length), the resulting asymmetry is felt by the metallic states extending over the film thickness, even though the substrate and surface potential are screened in the film interior. In this case, as we argue in this Rapid Communication, the band structure changes throughout the film and the Dyakonov-Perel (DP) mechanism [17] for spin dephasing is activated and becomes the dominant cause of spin reduction. The DP mechanism (that we briefly describe below) is known to be important in III-V or II-VI semiconductors or semiconductor heterostructures due to their inversion asymmetry $[18,19]$, but, to our knowledge, it has been largely overlooked so far in the important case of supported films or metallic bilayers, which show manifestly no inversion symmetry. Only recently, data from spin-pumping [20] and weak antilocalization [21] experiments in ultrathin films were found to fit the DP and not the EY mechanism.

Characteristic of systems with spin-orbit coupling and timereversal symmetry but broken inversion symmetry is the lifting of conjugation degeneracy [11] at each crystal momentum $\boldsymbol{k}$ and energy $E_{k}$ of the band structure. The resulting pair of states $\Psi_{k}^{ \pm}$obtains energies $E_{k}^{ \pm}$with the (usually small) splitting $\hbar\left|\boldsymbol{\Omega}_{\boldsymbol{k}}\right|=E_{\boldsymbol{k}}^{+}-E_{\boldsymbol{k}}^{-}$depending on the spin-orbit strength, the strength of the antisymmetric part of the potential $V_{\mathrm{A}}$, and the overlap of the wave function $\Psi_{k}$ with $V_{\mathrm{A}}$. The situation is

\footnotetext{
*h.nguyen@fz-juelich.de

†ph.mavropoulos@fz-juelich.de

†y.mokrousov@fz-juelich.de
}

described by adding to the $\boldsymbol{k}$-dependent crystal Hamiltonian the term

$$
\Delta H(\boldsymbol{k})=\frac{\hbar}{2} \boldsymbol{\Omega}_{\boldsymbol{k}} \cdot \boldsymbol{\sigma},
$$

where $\sigma$ is the vector of Pauli matrices and the vector quantity $\boldsymbol{\Omega}_{\boldsymbol{k}}$ is called the spin-orbit field (SOF) [8,22]. The direction of $\pm \boldsymbol{\Omega}_{\boldsymbol{k}}$ is given by the direction of the spin expectation value of $\Psi_{k}^{ \pm}$.

From this well-known theory follows the Dyakonov-Perel mechanism of spin dephasing. In brief, one assumes that an electron wave packet at wave vector $\boldsymbol{k}$ with a given spin direction $s_{k}$ is composed of a superposition of $\Psi_{k}^{ \pm}$. Then, effectively, $\boldsymbol{\Omega}_{\boldsymbol{k}}$ will act on the spin as a magnetic field due to Eq. (1) and $\boldsymbol{s}_{\boldsymbol{k}}$ will precess around $\boldsymbol{\Omega}_{\boldsymbol{k}}$. After an average momentum lifetime $T_{\mathrm{p}}$, the electron is scattered with a transition rate $P_{\boldsymbol{k}^{\prime} \boldsymbol{k}}$ to $\boldsymbol{k}^{\prime}$ occupying a superposition of $\Psi_{\boldsymbol{k}^{\prime}}^{ \pm}$ (the scattering is assumed to be energy and spin conserving) and precesses around $\boldsymbol{\Omega}_{\boldsymbol{k}^{\prime}}$, etc. Since the scattering sequence is a stochastic process, the electron spin effectively precesses around a sequence of random axes and the information on the initial direction of $s_{\boldsymbol{k}}$ is finally lost after a characteristic Dyakonov-Perel time of $T_{\mathrm{DP}}$. The process is governed by a kinetic equation [22]:

$$
\frac{\partial s_{k}}{\partial t}=\boldsymbol{\Omega}_{\boldsymbol{k}} \times \boldsymbol{s}_{\boldsymbol{k}}-\sum_{\boldsymbol{k}^{\prime}} P_{\boldsymbol{k}^{\prime} \boldsymbol{k}}\left(\boldsymbol{s}_{\boldsymbol{k}}-\boldsymbol{s}_{\boldsymbol{k}^{\prime}}\right) .
$$

If the splitting $\hbar\left|\boldsymbol{\Omega}_{\boldsymbol{k}}\right|$ is too large, then the scattering amplitude of the two functions $\Psi_{k}^{ \pm}$is also largely different. In that case, the semiclassical DP picture breaks down, because a single wave packet, initially occupying $\Psi_{k}^{ \pm}$simultaneously, will not be able to follow the scattering path in $\boldsymbol{k}$ space over several scattering events. In this sense, we expect that the Rashba surface states of metals, being in many cases characterized by a large $\hbar\left|\boldsymbol{\Omega}_{\boldsymbol{k}}\right|$ (e.g., of the order of $100 \mathrm{meV}$ at the Fermi level for $\mathrm{Au}(111)[23,24])$, will produce strong spin relaxation but not follow the DP mechanism.

In the present Rapid Communication we demonstrate the importance of the DP mechanism in metallic films deposited on insulating substrates. We use the density-functional-based Korringa-Kohn-Rostoker Green's function method for the calculation of the band structure and transition rates [25-28], and Eq. (2) for the time evolution of the spin expectation value. 
In our calculations we explicitly assume that all scattering is caused by self-adatom impurities, which are always present on metal surfaces; the concept that we demonstrate, however, is valid also in the presence of other scattering sources and can be easily quantified if the transition rate is known. As we find, the dephasing process is controlled by an interplay between film thickness, scattering strength, and penetration depth of the film wave functions into the insulating substrate and into the vacuum.

In the following, we give a short description of our method of calculation of spin-orbit fields, focusing on the basic principles and the approximations [29]. In a metallic film of thickness $d$ deposited on an insulating substrate, the wave functions $\Psi_{k}$ around the Fermi energy probe the surface and substrate potential, $V_{\text {surf }}$ at $z>d / 2$ and $V_{\text {sub }}$ at $z<-d / 2$, only by exponentially evanescent tails $(z=0$ defines the film midplane). Since by assumption, the freestanding film shows inversion symmetry, the antisymmetric part of the potential is just $V_{\mathrm{A}}(\boldsymbol{r})=\frac{\operatorname{sgn}(z)}{2}\left[V_{\text {surf }}(\boldsymbol{r})-V_{\text {sub }}(-\boldsymbol{r})\right]$. The smallness of the overlap $\left(\Psi_{k}, V_{\mathrm{A}} \Psi_{k}\right) \propto 1 / d$ allows us to calculate $\boldsymbol{\Omega}_{\boldsymbol{k}}$ in linear response to $V_{\mathrm{A}}$ with the freestanding film as a reference (the linear approximation improving at larger thicknesses). In a second, simplifying step, the substrate is mimicked by a constant barrier $V_{0}$ added to the surface potential of the freestanding film at $z<-d / 2$, yielding $V_{\mathrm{A}}=-\frac{\operatorname{sgn}(z)}{2} V_{0} \theta(|z|-d / 2)$. The conceptual advantage of this approximation is that one can define spin-orbit fields characteristic of the freestanding film, where the substrate enters only via a linear multiplicative factor $V_{0}$. That is, one obtains the linear relation

$$
\boldsymbol{\Omega}_{\boldsymbol{k}}=V_{0} \boldsymbol{\omega}_{k},
$$

where the spin-orbit field susceptibility $\omega_{\boldsymbol{k}}$ was introduced. The value of $\omega_{k}$ can be calculated in linear response theory on the basis of the freestanding film, while the parameter $V_{0}$ can be fitted at high symmetry points in the Brillouin zone for any given susbtrate with respect to an explicit calculation of the film on the substrate. We consider this second step well suited for a qualitative discussion, while the exact values of $\boldsymbol{\Omega}_{\boldsymbol{k}}$ can deviate somewhat from this result. Quantitative improvements by taking the full substrate potential explicitly into account are possible but numerically expensive and are not necessary to unravel the general phenomenon which is the motivation here.

In solving Eq. (2) for the spin population $s_{k}(t)$, we assume that the excited electron concentration is small, so that the scattering is practically not affected by the final state occupation, and that the excited states are close to the Fermi level, confining in practice the $\boldsymbol{k}$ values to the Fermi surface (FS). As an initial condition at $t=0$, we choose that $s_{k}$ is along the positive $z$ axis, i.e., normal to the film surface. Other choices of initial conditions, corresponding to different physical situations, are of course possible. A Fermi surface integration gives us the sought-for quantity $\left\langle s_{z}(t)\right\rangle=\left(1 / n_{\mathrm{F}}\right) \sum_{k}\left[s_{k}(t)\right]_{z}$, i.e., the magnetization along the initial axis $z$, normalized to the density of states $n_{\mathrm{F}}$ at the Fermi level. We assume that we are in the low concentration regime, i.e., $P_{\boldsymbol{k} \boldsymbol{k}^{\prime}}$ scales linearly to the impurity concentration. Even under this assumption, the solution of Eq. (2) has no simple scaling properties with respect to concentration or to
$V_{0}$. Equation (2) has to be explicitly solved for each set of these parameters.

We also compare the DP with the EY mechanism that neglects precession but accounts for spin-flip scattering. We employ the master equation for the spin-dependent electron distribution function $n_{k}^{\sigma}(t)$ involving spin-conserving and spinflip transition rates $P_{\boldsymbol{k} \boldsymbol{k}^{\prime}}^{\sigma \sigma^{\prime}}$ [29],

$$
\frac{d n_{\boldsymbol{k}}^{\sigma}}{d t}=\sum_{\boldsymbol{k}^{\prime}}\left[P_{\boldsymbol{k}^{\prime}}^{\sigma \sigma} n_{\boldsymbol{k}^{\prime}}^{\sigma}+P_{\boldsymbol{k}^{\prime}}^{\sigma \bar{\sigma}} n_{\boldsymbol{k}^{\prime}}^{\bar{\sigma}}-P_{\boldsymbol{k}^{\prime} \boldsymbol{k}}^{\sigma \sigma} n_{\boldsymbol{k}}^{\sigma}-P_{\boldsymbol{k}^{\prime} \boldsymbol{k}}^{\bar{\sigma} \sigma} n_{\boldsymbol{k}}^{\sigma}\right],
$$

where $\bar{\sigma}=\uparrow$ if $\sigma=\downarrow$ and vice versa. A time integration gives $\left\langle s_{z}(t)\right\rangle=\frac{\hbar}{2} \frac{1}{n_{F}} \sum_{k}\left[n_{k}^{\uparrow}(t)-n_{k}^{\downarrow}(t)\right]$. Contrary to Eq. (2), Eq. (4) is linear in the impurity concentration.

As we find, the form of $\left\langle s_{z}(t)\right\rangle$ is rather complicated, not having an exponential envelope. Still, we define the dephasing time $T_{\mathrm{DP}}$ and relaxation time $T_{\mathrm{EY}}$ as the time at which $\langle s(t)\rangle=\exp (-1)\langle s(0)\rangle$. Especially the EY mechanism causes an initial exponential decay with decay parameter $1 / T_{\mathrm{EY}}=2 \sum_{\boldsymbol{k} \boldsymbol{k}^{\prime}} P_{\boldsymbol{k} \boldsymbol{k}^{\prime}}^{\downarrow \uparrow} / n_{\mathrm{F}}$. In reality, both mechanisms act simultaneously, which should be taken into account in a full theory [30]. Here, however, we are after a separation of causes, comparing the two mechanisms as if they were independent.

We proceed with a presentation of our results. In Fig. 1 we show the Fermi surface distribution of the spin-orbit field susceptibility for $\mathrm{Au}(111)$ and $\mathrm{Pt}(111)$ 6-layer and 12-layer films (omitting surface states). The arrows denote the direction
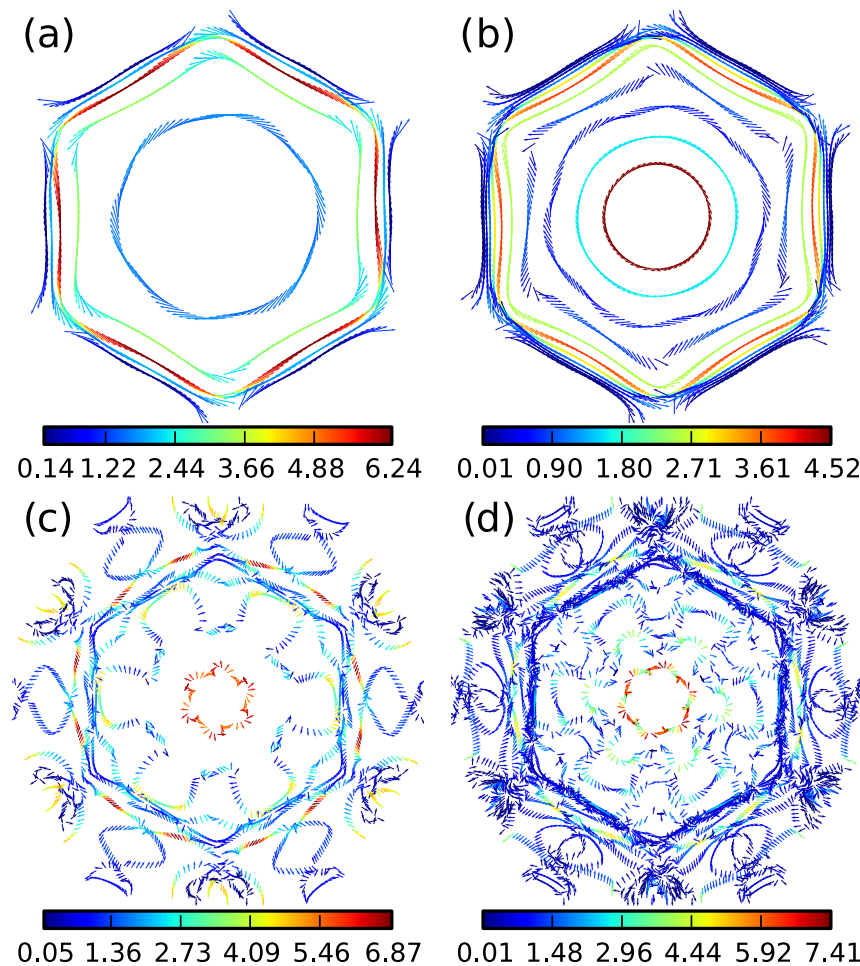

$0.051 .362 .734 .095 .46 \quad 6.87$

FIG. 1. The distribution of the spin-orbit field susceptibility $\omega_{k}$ on the Fermi surfaces of (a) 6-layer $\mathrm{Au}(111)$, (b) 12-layer $\mathrm{Au}(111)$, (c) 6-layer Pt(111), and (d) 12-layer Pt(111) films. The arrows denote the projection of the direction of the spin-orbit fields onto the surface plane. The color code denotes the absolute value of $\hbar\left|\boldsymbol{\omega}_{\boldsymbol{k}}\right| \times 10^{3}$. The surface states are not shown. See the Supplemental Material for plots with improved resolution [29]. 


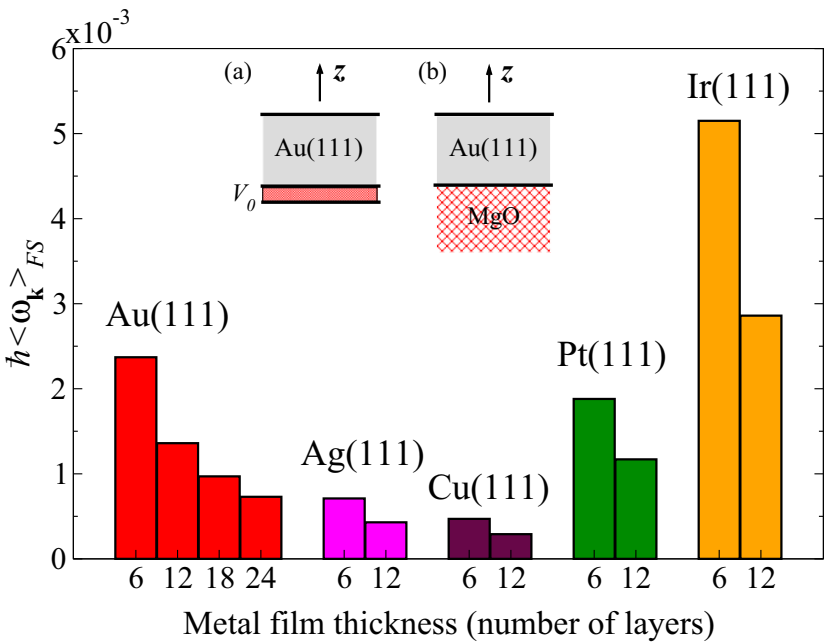

FIG. 2. The Fermi surface average of spin-orbit field susceptibility $\hbar\langle|\omega|\rangle$ in various metallic films as a function of film thickness. The inset shows the $\mathrm{Au}(111)$ film (a) on the model substrate $V_{0}$ and (b) on the $\mathrm{MgO}$ substrate.

of the $\omega_{k}$ projected onto the surface plane, while the color code shows the value of $\hbar\left|\omega_{k}\right| \times 10^{3}$. We find sizable out-of-plane components of $\omega_{k}$ [29]. These must identically vanish only in the presence of in-plane inversion symmetry, $V(x, y, z)=$ $V(-x,-y, z)$ [31], e.g., in bcc/fcc(100) or (110) systems.

The magnitude of the spin-orbit field susceptibility shows a spread, as seen from the color code. Averaging over the Fermi surface, we expect $\langle|\omega|\rangle \propto 1 / d$. Qualitatively, this behavior is indeed observed in Fig. 2 for all tested systems: Au(111), $\mathrm{Ag}(111), \mathrm{Cu}(111), \operatorname{Pt}(111)$, and $\operatorname{Ir}(111)$ [32]. Comparing $\mathrm{Au}(111), \operatorname{Ag}(111)$, and $\mathrm{Cu}(111)$ at the same thickness, we find that stronger spin-orbit coupling leads to larger averaged SOF, as expected from a spin-orbit phenomenon.

For an estimation of the parameter $V_{0}$ [Eq. (3)] we calculated self-consistently a $6 \mathrm{ML} \mathrm{Au(111)} \mathrm{film} \mathrm{on} \mathrm{six} \mathrm{layers} \mathrm{of} \mathrm{the}$ wide-band-gap insulator $\mathrm{MgO}$ and found explicitly the SOF at high symmetry points in the Brillouin zone. A fit to $V_{0} \omega_{k}$, with $\omega_{k}$ calculated for a freestanding six-atomic-layer $\mathrm{Au}(111)$ film, gives us $V_{0}=-12.24 \mathrm{eV}$, which we accept as characteristic of the $\mathrm{Au} / \mathrm{MgO}$ interface at all film thicknesses. Thus the Fermi surface average yields spin-orbit field of $\hbar\langle|\boldsymbol{\Omega}|\rangle=29 \mathrm{meV}$ [for six layers of $\operatorname{Pt}(111)$ we obtain $\hbar\langle|\boldsymbol{\Omega}|\rangle=25 \mathrm{meV}$ ]. The value decreases to $9 \mathrm{meV}$ in a 24-atomic-layer $\mathrm{Au}(111)$ film, which is still considerably larger than a typical value of $1 \mathrm{meV}$ met in semiconductors [8], with the consequence of a much faster spin precession in these metallic films (a splitting of $\hbar|\boldsymbol{\Omega}|=1 \mathrm{meV}$ corresponds to a Larmor precession time of $T_{\mathrm{L}}=4.13 \mathrm{ps}$ ). Different insulating substrates are expected to have different values of $V_{0}$ but in the same order of magnitude.

Now we are ready to discuss the solution of the kinetic equation (2). Figure 3 shows the value of $\left\langle s_{z}(t)\right\rangle /\left\langle s_{z}(0)\right\rangle$ for a 24-atomic-layer $\mathrm{Au}(111)$ film. The adatom concentration is set to $1 \%$. The initial rapid drop gives $T_{\mathrm{DP}} \sim 0.1-0.3 \mathrm{ps}$. The behavior is clearly oscillatory with a nonexponential envelope and with part of the signal ( $\sim 2 \%$ of the initial value) persisting to times as large as $80 \mathrm{ps}$, as can be seen from the inset of Fig. 3. We also explored a variation of the parameter $V_{0}$ to

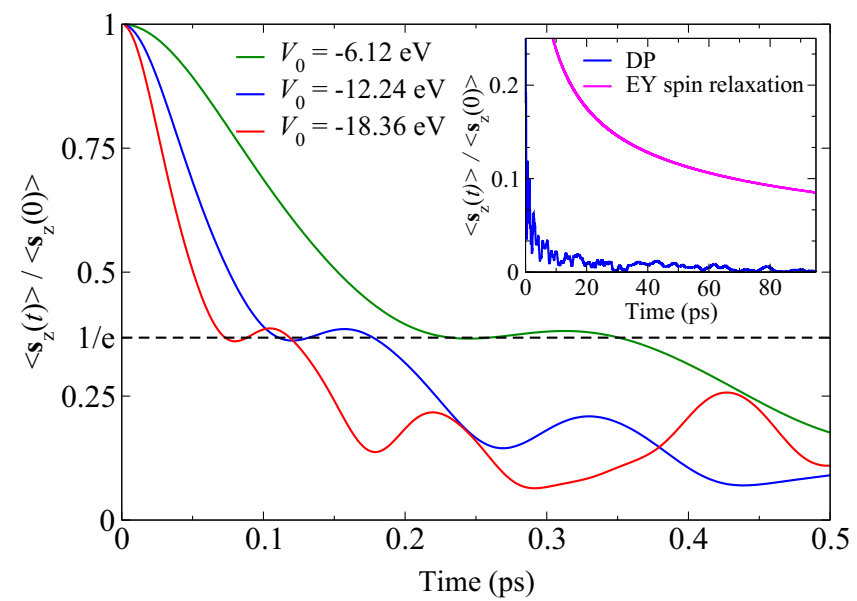

FIG. 3. The Dyakonov-Perel time decay of the total electron spin in 24-layer $\mathrm{Au}$ (111) films with $1 \%$ adatom impurities. Three cases are considered: $V_{0}=-6.12,-12.24$, and $-18.36 \mathrm{eV}$. Inset: the case of $V_{0}=-12.24 \mathrm{eV}$ on a larger time scale, together with the Elliott-Yafet decay.

-6.12 and $-18.36 \mathrm{eV}$; the qualitative behavior is the same, but with a slower or faster decay, respectively, due to the different precession frequency (see Fig. 3).

The origin of this behavior lies in the standing-wave nodal structure that the metal wave functions ("quantum-well states") exhibit in the $z$ direction (perpendicular to the film). Depending on the band, $\left|\Psi_{k}(\boldsymbol{r})\right|^{2}$ can have a negligible value in the vacuum and in the substrate, in which case $\boldsymbol{\Omega}_{\boldsymbol{k}} \sim$ $\left(\Psi_{k}, V_{\mathrm{A}} \Psi_{k}\right)$ almost vanishes. At these $\boldsymbol{k}$ points, the precession term in Eq. (2) is negligible even over long times. The only path to dephasing for these spins is to be scattered away first to some $\boldsymbol{k}^{\prime}$ with larger SOF. But since the initial state $\Psi_{\boldsymbol{k}}$ does not penetrate in the vacuum, the overlap with the adatom potential is also small, keeping the scattering rate low. These particular parts of the FS act in a sense as spin traps. Since the nodal structure of the quantum-well states depends primarily on the metallic film and not on the substrate or on the adatom, the spin traps are a property of the pristine film. However, in the presence of buried impurities instead of adatoms, or of phonons at elevated temperatures, the scattering rate will not be negligible and the spins will be scattered away from the traps at a higher rate. Additionally, the precession can freeze if $\boldsymbol{\Omega}_{\boldsymbol{k}}$ and $\boldsymbol{s}_{\boldsymbol{k}}$ are collinear. Since this condition is met in part of the FS (not shown) of $\mathrm{Au}(111)$ or $\mathrm{Pt}(111)$, it is part of the reason for the slow decay of $s_{z}$ in these systems. We should note that the existence and effectiveness of spin traps is material and thickness dependent. Figure 3 (inset) also shows $\left\langle s_{z}(t)\right\rangle /\left\langle s_{z}(0)\right\rangle$ by the EY mechanism [Eq. (4)]. Evidently, the EY spin relaxation is also affected by the spin traps, producing persisting tails, and is not exponential at large times. It is clear, however, that the DP mechanism dominates the decay process.

Table I summarizes the values of $T_{\mathrm{DP}}$ for 6-atomic-layer and 24-atomic-layer $\mathrm{Au}(111)$ with $1 \%$ and $5 \%$ of self-adatoms and for different substrate-potential values $V_{0}$. The same table also shows the calculated EY relaxation time, including values with ("ws") and without ("wos") the surface states taken into account. (The latter serves for comparing the DP and 
TABLE I. Spin-depasing time induced by the Dyakonov-Perel mechanism, $T_{\mathrm{DP}}$, in comparison to the spin-relaxation time $T_{\mathrm{EY}}$ induced by the Elliott-Yafet mechanism and the momentum-relaxation time $T_{\mathrm{p}}$ in 6-layer $\mathrm{Au}(111)$ and 24-layer $\mathrm{Au}(111)$ films with $\mathrm{Au}$ adatoms as scatterers. The adatom concentration is taken with respect to full surface coverage. $T_{\mathrm{EY}}$ and $T_{\mathrm{p}}$ are given with (ws) and without (wos) the surface states taken into account.

\begin{tabular}{|c|c|c|c|c|c|c|c|}
\hline \multirow[b]{2}{*}{$V_{0}(\mathrm{eV})$} & \multicolumn{3}{|c|}{$T_{\mathrm{DP}}(\mathrm{ps})$} & \multicolumn{2}{|c|}{$T_{\mathrm{EY}}(\mathrm{ps})$} & \multicolumn{2}{|c|}{$T_{\mathrm{p}}(\mathrm{ps})$} \\
\hline & -6.12 & -12.24 & -18.36 & ws & wos & ws & wos \\
\hline 6-layer, $1 \%$ imp. & 0.054 & 0.027 & 0.018 & 5.84 & 16.06 & 0.10 & 0.51 \\
\hline 6-layer, $5 \%$ imp. & 0.059 & 0.029 & 0.018 & 1.16 & 3.20 & 0.02 & 0.10 \\
\hline 24-layer, $1 \%$ imp. & 0.22 & 0.11 & 0.072 & 1.27 & 47.65 & 0.56 & 1.06 \\
\hline 24-layer, 5\% imp. & 0.31 & 0.17 & 0.083 & 0.25 & 9.53 & 0.11 & 0.21 \\
\hline
\end{tabular}

EY mechanisms acting on the same set of states.) As is qualitatively expected [8], $T_{\mathrm{DP}}$ increases and $T_{\mathrm{EY}}$ is reduced with increasing defect concentration. It is striking that $T_{\mathrm{DP}}$ is in all cases much lower than $T_{\mathrm{EY}}$. The reason for this is basically the very high value of the spin-orbit fields causing a Larmor precession period $T_{\mathrm{L}}$ that is smaller than the momentum relaxation time $T_{\mathrm{p}}$. For example, for 24 layers of $\mathrm{Au}(111)$, $\langle|\boldsymbol{\Omega}|\rangle=9 \mathrm{meV}$ giving $T_{\mathrm{L}}=0.46 \mathrm{ps}$, to be compared to $T_{\mathrm{p}} \sim$ $1 \mathrm{ps}$ at $1 \%$ adatom concentration. In this regime, according to Zutic et al. [8], $T_{\mathrm{DP}}$ is estimated as the inverse of the SOF spread $\Delta \Omega$. For 24 layers of $\mathrm{Au}(111), T_{\mathrm{DP}} \sim 1 / \Delta \Omega=67$ fs, which is in the same order as our first-principles value of $T_{\mathrm{DP}}=110 \mathrm{fs}$. Only at much larger thicknesses does the average precession period exceed a few ps, allowing the EY mechanism to dominate [33]. Assuming that $\langle|\boldsymbol{\Omega}|\rangle \propto 1 / d$, we can estimate that the two mechanisms will have a comparable contribution at thicknesses of approximately 270 layers $(70 \mathrm{~nm})$.

The SOF could be washed out at elevated temperatures. Phonons and electron-electron scattering, or strong bulk disorder, may reduce the electron phase relaxation length to values smaller than the film thickness, so that electron states in the film interior cannot probe the substrate-surface asymmetry (different from semiconductors, the asymmetry potential does not penetrate deep into the metallic film interior due to metallic screening). Strong bulk scattering may also promote the EY mechanism and freeze-out the DP mechanism by motional narrowing. In cases of spin valves, when the metallic film is sandwiched between ferromagnetic layers, inversion symmetry is restored and the SOF vanish. In cases of weak spin-orbit coupling, e.g., $\mathrm{Li}, \mathrm{Na}, \mathrm{Mg}$, or $\mathrm{Al}$, both the SOF and the spin mixing of states are reduced proportionally to the spin-orbit strength. As a result the DP mechanism is still expected to dominate at small thicknesses, however, it is expected to enter the motional narrwowing regime because of the slower Larmor precession [8].

Finally, one expects an anisotropy of the DP dephasing time with respect to the initial condition, e.g., $T_{\mathrm{DP}}$ will be different for $s_{k}(t=0)$ perpendicular to the film compared to its being in the film plane. This type of anisotropy has been reported previously, e.g., in semiconductor heterostructures or in graphene [34], and originates from a different microscopic mechanism compared to the one reported for inversionsymmetric metals [35-37] or phosphorene [38,39]. Using the symbol $\hat{s}$ to denote the direction of the initial spin polarization, we have a dependence $T_{\mathrm{DP}}(\hat{s})$ and we may define the anisotropy as the relative difference

$$
\mathcal{A}_{\mathrm{DP}}=\frac{\max _{\hat{s}} T_{\mathrm{DP}}(\hat{s})-\min _{\hat{s}} T_{\mathrm{DP}}(\hat{s})}{\min _{\hat{s}} T_{\mathrm{DP}}(\hat{s})} .
$$

For the films studied here, $\mathcal{A}_{\mathrm{DP}}$ reaches values as large as $200 \%$ in a 24-atomic-layer $\mathrm{Au}(111)$ film. The spin traps are persistent irrespective of the initial condition, as they originate in regions of very small SOF, irrespective of the $\hat{s}$.

In conclusion, we find that the potential asymmetry introduced in supported metallic films by the substrate will create strong spin-orbit fields, activating the DP mechanism of spin dephasing. This effect is present in spite of the strong charge screening in metals, since the surface and substrate are probed by the itinerant metal wave functions even if the potential perturbation vanishes in the film interior. We predict that the DP mechanism can dominate over the EY mechanism for thicknesses as large as 200-300 atomic layers, after which the interface induced SOF become very small, falling off inversely proportional with the thickness of the film. In the case of scattering only by adatoms, certain parts of the Fermi surface with low SOF acquire also low scattering rates, acting as spin traps and allowing the spin signal to persist over long times.

Our theory is corroborated by recent experiments in ultrathin films $[20,21]$. We propose that an experiment to verify our predictions can be based on laser-pulse, pumpprobe experiments (probing the Faraday or Kerr rotation) with femtosecond temporal resolution in films of varying thickness $[40,41]$.

We would like to thank Gustav Bihlmayer, Jaroslav Fabian, and Swantje Heers for fruitful discussions. This work was financially supported by Deutsche Forschungsgemeinschaft Projects No. MO 1731/3-1 and No. SPP 1538 SpinCaT, and the HGF-YIG NG-513 project of the Helmholtz Gemeinschaft. We acknowledge computing time on the supercomputers JUQEEN and JUROPA at Jülich Supercomputing Center and JARA-HPC Compute cluster of RWTH Aachen University.
[1] F. J. Jedema, A. T. Filip, and B. J. van Wees, Nature London 410, 345 (2001).

[2] J. Sinova, D. Culcer, Q. Niu, N. A. Sinitsyn, T. Jungwirth, and A. H. MacDonald, Phys. Rev. Lett. 92, 126603 (2004).

[3] S. O. Valenzuela and M. Tinkham, Nature (London) 442, 176 (2006).

[4] T. Kimura, Y. Otani, T. Sato, S. Takahashi, and S. Maekawa, Phys. Rev. Lett. 98, 156601 (2007).
[5] T. Saeki, Y. Hasegawa, S. Mitani, S. Takahashi, H. Imamura, S. Maekawa, J. Nitta, and K. Takanashi, Nat. Mater. 7, 125 (2008).

[6] S.-G. Cheng, Y. Xing, Q.-F. Sun, and X. C. Xie, Phys. Rev. B 78, 045302 (2008).

[7] K. Tauber, M. Gradhand, D. V. Fedorov, and I. Mertig, Phys. Rev. Lett. 109, 026601 (2012).

[8] I. Zutic, J. Fabian, and D. Sarma, Rev. Mod. Phys. 76, 323 (2004). 
[9] J. Fabian and S. Das Sarma, Phys. Rev. Lett. 81, 5624 (1998).

[10] R. J. Elliott, Phys. Rev. 96, 266 (1954).

[11] Y. Yafet, in Solid State Physics, edited by F. Seitz and D. Turnbull (Academic, NewYork, 1963), Vol. 14, p. 1.

[12] D. Lubzens and S. Schultz, Phys. Rev. Lett. 36, 1104 (1976).

[13] M. Johnson and R. H. Silsbee, Phys. Rev. Lett. 55, 1790 (1985).

[14] M. Gradhand, M. Czerner, D. V. Fedorov, P. Zahn, B. Y. Yavorsky, L. Szunyogh, and I. Mertig, Phys. Rev. B 80, 224413 (2009).

[15] M. Gradhand, D. V. Fedorov, P. Zahn, and I. Mertig, Phys. Rev. B 81, 020403(R) (2010).

[16] The EY mechanism concerns spin-flip scattering events due to the spin-mixed character of Bloch states in the presence of spin-orbit coupling.

[17] M. I. D’yakonov and V. I. Perel', Fiz. Tverd. Tela 13, 3581 (1971) [Sov. Phys. Solid State 13, 3023 (1972)].

[18] G. E. Pikus and A. N. Titkov, in Optical Orientation, edited by F. Meier and B. P. Zakharchenya (North Holland, Amsterdam, 1984), pp. 73-131.

[19] M. D. Mower, G. Vignale, and I. V. Tokatly, Phys. Rev. B 83, 155205 (2011).

[20] C. T. Boone, J. M. Shaw, H. T. Nembach, and T. J. Silva, J. Appl. Phys. 117, 223910 (2015).

[21] J. Ryu, M. Kohda, and J. Nitta, Phys. Rev. Lett. 116, 256802 (2016).

[22] J. Fabian, A. Matos-Abiague, C. Ertler, P. Stano, and I. Zutic, Acta Phys. Slov. 57, 565 (2007).

[23] S. LaShell, B. A. McDougall, and E. Jensen, Phys. Rev. Lett. 77, 3419 (1996).

[24] J. Henk, A. Ernst, and P. Bruno, Phys. Rev. B 68, 165416 (2003).

[25] S. Heers, P. Mavropoulos, S. Lounis, R. Zeller, and S. Blügel, Phys. Rev. B 86, 125444 (2012).

[26] N. H. Long, P. Mavropoulos, B. Zimmermann, S. Heers, D. S. G. Bauer, S. Blügel, and Y. Mokrousov, Phys. Rev. B 87, 224420 (2013).

[27] N. H. Long, P. Mavropoulos, B. Zimmermann, D. S. G. Bauer, S. Blügel, and Y. Mokrousov, Phys. Rev. B 90, 064406 (2014).
[28] B. Zimmermann, P. Mavropoulos, N. H. Long, C.-R. Gerhorst, and S. Blügel, and Y. Mokrousov, Phys. Rev. B 93, 144403 (2016).

[29] See Supplemental Material at http://link.aps.org/supplemental/ 10.1103/PhysRevB.94.180406 for a detailed discussion.

[30] P. Boross, B. Dora, A. Kiss, and F. Simon, Sci. Rep. 3, 3233 (2013).

[31] This is because the in-plane reflection operator $R_{x y}$ transforms $\Psi_{k}$ to $\Psi_{-k}$ which is also the time-reverse state; thus the $z$ component of the spin, $s_{z}$, must flip by time reversal. At the same time, $R_{x y} \sigma_{z} R_{x y}^{-1}=+\sigma_{z}$, i.e., $s_{z}$ must be conserved. This leads to $s_{z}=0$, i.e., also $\left(\boldsymbol{\Omega}_{\boldsymbol{k}}\right)_{z}=0$.

[32] This trend can be violated at specific $\boldsymbol{k}$ points, as was shown, e.g., for Pb films on Si [see. J. H. Dil, F. Meier, J. Lobo-Checa, L. Patthey, G. Bihlmayer, and J. Osterwalder, Phys. Rev. Lett. 101, 266802 (2008)].

[33] $T_{\mathrm{p}}$ is, to a first approximation, independent of the film thickness: the scattering matrix element off impurities scales as $\propto 1 / d$, but the number of available final states increase as $\propto d$.

[34] S. Fratini, D. Gosálbez-Martinez, P. Merodio Cámara, and J. Fernańdez-Rossier, Phys. Rev. B 88, 115426 (2013).

[35] B. Zimmermann, P. Mavropoulos, S. Heers, N. H. Long, S. Blügel, and Y. Mokrousov, Phys. Rev. Lett. 109, 236603 (2012).

[36] Y. Mokrousov, H. Zhang, F. Freimuth, B. Zimmermann, N. H. Long, J. Weischenberg, I. Souza, P. Mavroupolos, and S. Blügel, J. Phys.: Condens. Matter 25, 163201 (2013).

[37] N. H. Long, P. Mavropoulos, S. Heers, B. Zimmermann, Y. Mokrousov, and S. Blügel, Phys. Rev. B 88, 144408 (2013).

[38] P. Li and I. Appelbaum, Phys. Rev. B 90, 115439 (2014).

[39] M. Kurpas, M. Gmitra, and J. Fabian, Phys. Rev. B 94, 155423 (2016).

[40] A. Y. Elezzabi, M. R. Freeman, and M. Johnson, Phys. Rev. Lett. 77, 3220 (1996).

[41] J. M. Kikkawa and D. D. Awschalom, Phys. Rev. Lett. 80, 4313 (1998). 